\title{
Research on Band Gap Algorithm and Regularity of Two Component Phononic Crystals
}

\author{
Jianning $\operatorname{Han}^{1 *}$, Shuai Tang ${ }^{2}$, Yu Liu ${ }^{1}$, Peng Yang ${ }^{1}$ and Lulu $\mathrm{Li}^{1}$ \\ ${ }^{1}$ School of Information and communication Engineering, North University of \\ China, Taiyuan 030051, China \\ ${ }^{2}$ School of Science, North University of China, Taiyuan 030051, China \\ 33161675@qq.com,442861228@qq.com
}

\begin{abstract}
The phononic crystal band calculation and control method were studied. We used the finite element software COMSOL to analyze the modulation law of band gap width. The material parameters, structure parameters and phononic crystal plate thickness were the main factors affecting the band gap. It was found that the larger the density ratio of the scattering body was, the greater the density of the matrix would be; the wider the band gap was, the greater the influence of the scattering body on the band gap appeared. In the aspect of structure parameters, it was found that the higher the symmetry was, the wider the bandgap width of the crystal lattice would be. In terms of filling rate, the forbidden band width became wider when the filling rate was increased, and the forbidden band width narrowed to a certain extent. In the aspect of phononic crystal plate thickness: twodimensional component phononic crystal plate thickness had an impact on the band gap. As the thickness of the plate increased, the band gap became wider. The results were of guiding significance for the design and fabrication of novel acoustic devices.
\end{abstract}

Keywords: Phononic crystals; Filter; Ultrasonic frequency; Finite element

\section{Introduction}

Phononic crystal is a new type of acoustic functional material with many acoustic properties that are not available in natural materials. In recent years, people have done a lot of research on phononic crystals. The propagation of acoustic waves in periodic composites is the main research direction of phononic crystals[1]-[5].The resonance characteristics and bandgap characteristics of phononic crystals can be obtained by the equations of motion of acoustic waves in phononic crystals. The significance of phononic crystal research is that these properties are used to control the propagation of sound waves in the crystal[6]-[8]. The formation of the band gap is mainly caused by multiple coherent scattering. The scattering mechanism is mainly divided into microscopic scattering and macroscopic scattering[9]-[12]. Microscopic scattering is mainly Mie scattering[13],[14]. The macroscopic scattering is mainly the Bragg scattering effect. The two scattering interactions form a band gap[15]-[17]. Various studies have shown that the formation of phononic crystal bandgap is mainly the influence of material parameters and structural parameters[17]-[20].

In this paper, the finite element method was adopted to calculate the bandgap of phononic crystal. The influence of component material parameters, structural parameters and plate thickness on the bandgap was discussed. The material parameters of the element included the matrix, the density of the scatterer, the modulus of elasticity and so on. The structural parameters included lattice form, filling rate and so on. In this paper, we mainly studied the influence of material parameters and filling rate on the bandgap, so as to

Received (October 29, 2017), Review Result (January 10, 2018), Accepted (January 15, 2018) 
determine the extent of the bandgap width affected by these factors, and provided theoretical guidance for the bandgap design of phononic crystals.

\section{Modulation Law Analysis based on Band Gap Width}

\subsection{Effect of Material Parameters on Structure of Phononic Crystal Bands}

The influence of the material parameters of the scattering body on the band gap was studied by fixing the remaining parameters and changing only one parameter. The phononic crystal model used is shown in Figure 1 . The fill rate $f=0.532$, lattice constant $a=0.0017 \mathrm{~m}$, radius of the scatterer cylinder $r=0.0007 \mathrm{~m}$. Scatter material was steel. The steel cylinders were regularly arranged in the epoxy resin by the square lattice. The related material parameters are shown in Table 1.

Table 1. The Related Material Parameters

\begin{tabular}{|c|c|c|c|}
\hline Materials & $\begin{array}{c}\text { Density } \\
\rho\left(k g \cdot m^{-3}\right)\end{array}$ & $\begin{array}{c}\text { Young modulus } \\
\text { E } \\
\text { (or Volume } \\
\text { modulusK)/ } \\
10^{10} \mathrm{~Pa} \\
\end{array}$ & $\begin{array}{l}\text { Shear modulus } \\
\qquad \mu / 10^{10} \mathrm{~Pa}\end{array}$ \\
\hline Epoxy resin & 1180 & 0.435 & 0.159 \\
\hline Gold & 19500 & 8.5 & 2.99 \\
\hline Tungsten & 19100 & 35.41 & 13.11 \\
\hline Lead & 11600 & 4.08 & 1.49 \\
\hline Copper & 8950 & 16.46 & 7.53 \\
\hline Steel & 7780 & 21.06 & 8.10 \\
\hline $\mathrm{Ti}$ & 4540 & 11.70 & 4.43 \\
\hline Aluminum & 2730 & 7.76 & 2.87 \\
\hline Carbon & 1750 & 23.01 & 8.85 \\
\hline Barium carbonate & 5300 & 10.6 & 4.09 \\
\hline Epoxy resin & 1180 & 0.435 & 0.159 \\
\hline
\end{tabular}

The finite element method was used to analyze the first six characteristic frequencies and modal shape of the phonon crystal structure. The results are shown in Table 2. In order to observe the characteristics of each feature frequency clearly, the mode shape is shown in Figure 1. 
Table 2. Phononic Crystal Structure of the Original Cell Frequency Table

\begin{tabular}{|c|c|c|c|c|c|c|}
\hline Order & (a) & (b) & (c) & (d) & (e) & (f) \\
\hline $\begin{array}{c}\text { Characteristic } \\
\text { Frequency } \\
(\mathrm{kHz})\end{array}$ & 236 & 323 & 388 & 411 & 499 & 510 \\
\hline
\end{tabular}

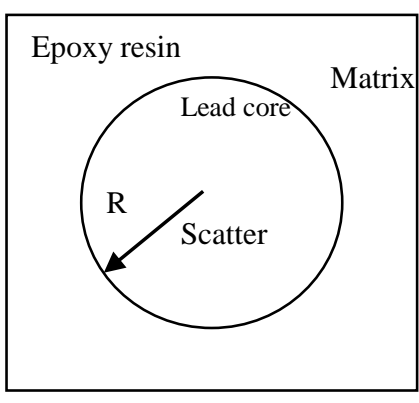

(a) The original cell diagram

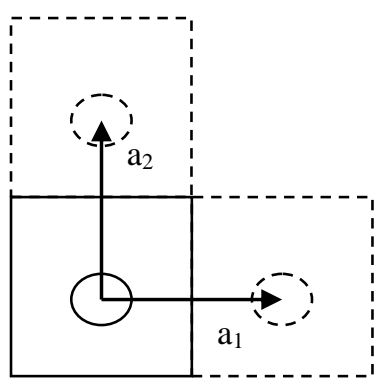

(b) The square lattice

\section{Figure 1. Structure of Two - dimensional Two - element Phononic Crystals}

It can be clearly seen from the vibration modes of the graphs of Figure 2.(a)、(b)、(c)、(d)、(e)、(f). For the graphs (a), (b), (c), (d), vibration mainly concentrates on the base of the steel cylinder scatterer. (a) and (b) form a narrow band gap of $236 \mathrm{kHz}$ to $323 \mathrm{kHz}$. For the (e) and (f) ,the vibration displacement is very small. In graphs (f), there are multiple peaks and valleys, that is, the energy is dispersed, so it is difficult to form a band gap.

When taking the scatterer densities from small to large of carbon, aluminum, steel, lead and gold (parameters shown in Table 1), We fixed other parameters. The filling rate $\mathrm{f}$ was $0.007 \mathrm{~m}$, and the lattice constant a was $0.0017 \mathrm{~m}$. The shape of the scatterer was circular, and the radius of the scatterer cylinder $\mathrm{r}=0.0007 \mathrm{~m}$. The phonon crystals formed by the material in the epoxy matrix were arranged in a square lattice cycle. And we studied the band structure of each material as a scatterer.

Table 3. Change the Band Gap Calculated by the Scatterer Material

\begin{tabular}{|c|c|c|c|c|c|}
\hline Material & carbon & $\begin{array}{c}\text { aluminu } \\
\mathrm{m}\end{array}$ & steel & lead & gold \\
\hline Bandgap & $\begin{array}{c}110 \sim 150 \\
\mathrm{kHz}\end{array}$ & $\begin{array}{c}900 \sim 130 \\
0 \mathrm{kHz}\end{array}$ & $\begin{array}{c}500 \sim 120 \\
0 \mathrm{kHz}\end{array}$ & $\begin{array}{c}419 \sim 599 \\
\mathrm{kHz}\end{array}$ & $\begin{array}{c}300 \sim 611 \\
0 \mathrm{kHz}\end{array}$ \\
\hline
\end{tabular}

The bandgap results of the phononic crystals of each material calculated by the finite element method are shown in Table 3. 


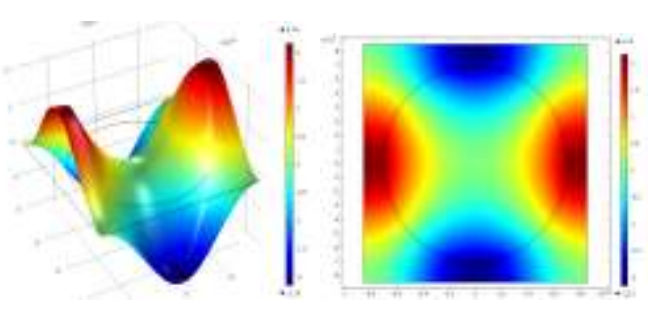

(a)

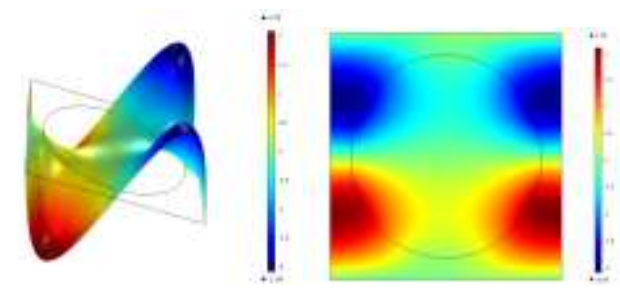

(c)

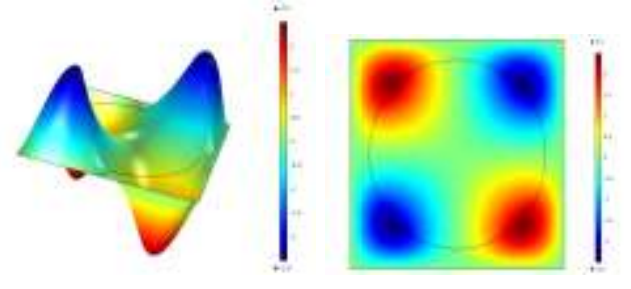

(e)

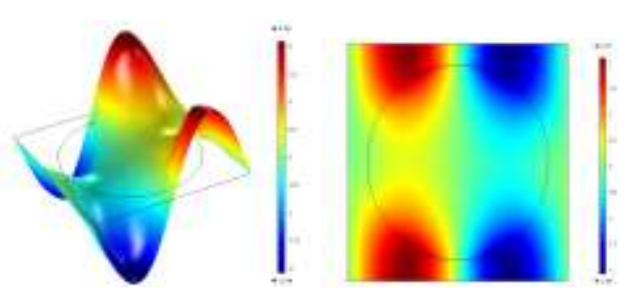

(b)

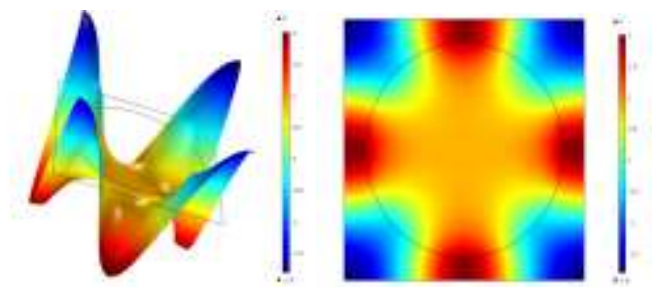

(d)

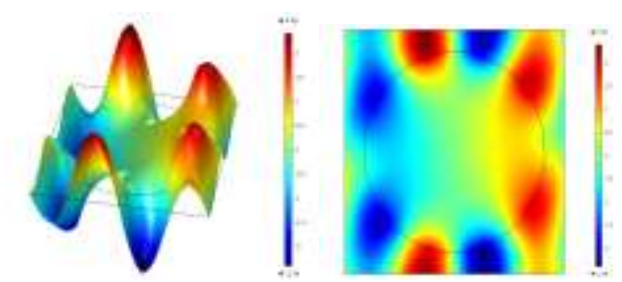

(f)

Figure 2. Finite Element Calculation of Phononic Crystals

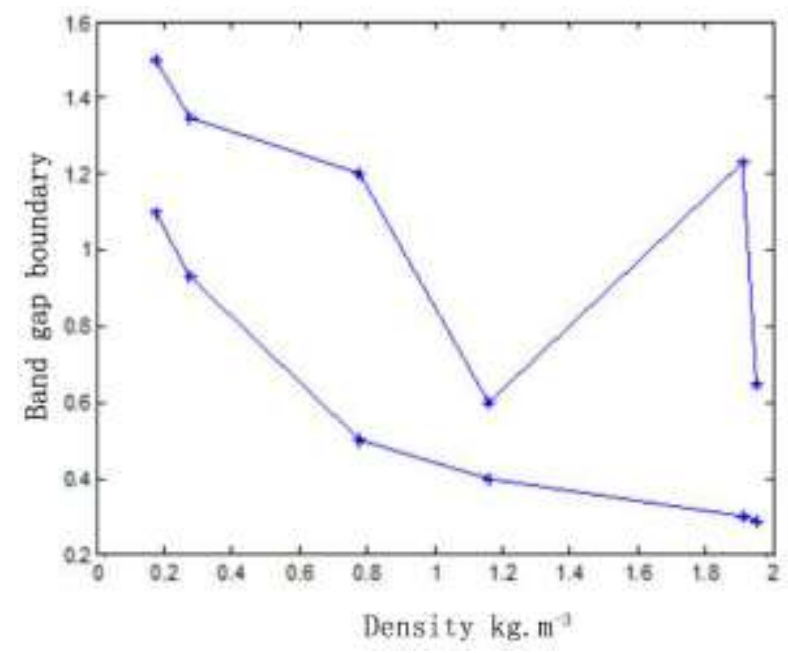

Figure 3. Variation of the Relative Width of the Band Gap with the Density of the Scatterer

It can be clearly seen in Figure 3, as the density of the scatterers increases, the lower boundary of the band gap moves downward (upper and lower curves represent the upper and lower boundaries, respectively). The change of band gap upper boundary is not large, but there are two inflection points. The two are lead and gold, respectively, and they all have in common that the Young's modulus is relatively small.

We discussed the effect of Young's modulus on the bandgap width. We took the Young's modulus from small to large of lead, aluminum, gold, barium carbonate, titanium, copper and steel, as shown in Figure 4. 


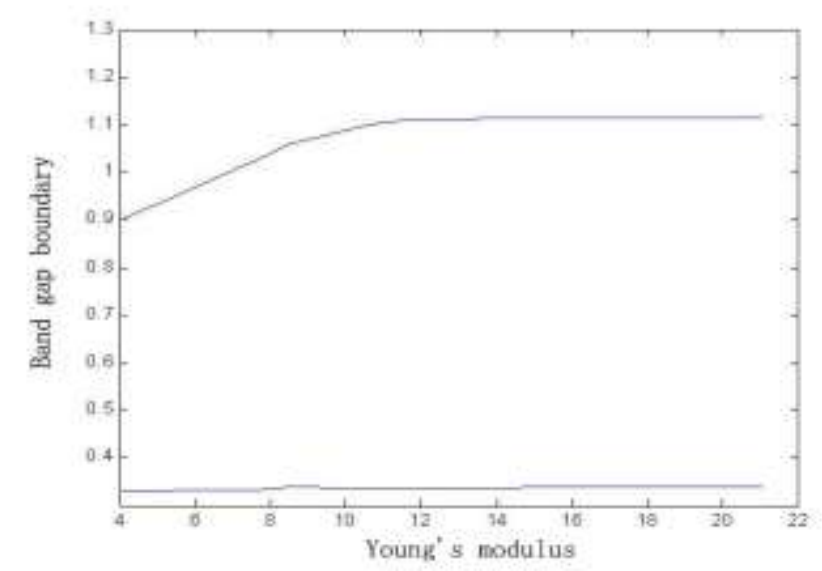

Figure 4. Variation of the Bandgap Boundary with Young's Modulus

It can be seen from the figure 4. that with the increase of the Young's modulus, the upper bound increases and the lower boundary of the bandgap changes little.

The effect of shear modulus on the band gap width is discussed below. We took lead, aluminum, gold, barium carbonate, titanium, copper, and steel sorted in ascending order of modulus of shear, and obtained a graph as shown in Figure 5 below.

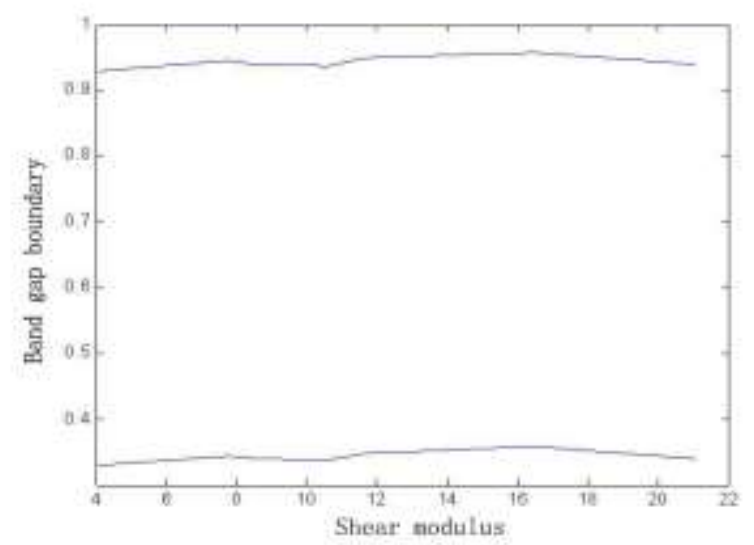

Figure 5. Variation of Band Gap Boundary with Shear Msodulus

It can be seen from Figure 5. that the upper and lower margins of the bandgap are not changed with the increase of the shear modulus.

The rule obtained from these three graphs is that the Young's modulus of the scatterer mainly affects the upper boundary of the bandgap. The larger the Young's modulus is, the more the upper edge of the bandgap moves. The density of the scatterer mainly affects the lower boundary of the bandgap. The greater the density is, the lower the lower gap of the bandgap will be. The shear modulus has little effect on the bandgap. Therefore, if a wide bandgap width is wanted, it is necessary to select the bigger scattering body density and the smaller corresponding Young's modulus of the material as a phononic crystal material.

The effect of changing the matrix material parameters on the bandgap is discussed below. Changed matrix material parameters, fixed lattice form, fill rate, scatterer material, and shape. The comparison shows that when the scatterer is determined, as the matrix density decreases, the lower bandgap decreases, which is similar to the decrease of scatterers' material density as the bandgap decreases. When the Young's modulus of the matrix increases, the width of the band gap do not change much. 
In summary, the density of the scattering material mainly affects the lower boundary of the bandgap. The greater the density is, the more the lower boundary of the bandgap moves down. The Young's modulus of the scatterer mainly affects the upper boundary of the bandgap. The larger the Young's modulus is, the more the upper boundary of the bandgap moves upwards. The influence of the density of the matrix material on the bandgap is similar to that of the scatterer, which only affects the lower boundary of the band gap and the Young's modulus of the matrix has little effect on the bandgap. The shear modulus has little effect on the band gap, compared with the density and Young's modulus could be ignored. Therefore, when the scatterer and the matrix material density difference is large, the band gap structure is wider.

\subsection{Influence of Structural Parameters on Gap Structure}

The influence of structural parameters on the bandgap mainly includes three aspects: lattice form, filling rate and scatterer shape. We used triangular lattice and square lattice as the lattice structure, and used elliptical scatterers and circular scatterers as the scatterers for further study.

\subsubsection{Influence of lattice form on the structure of phononic crystal}

We discussed the lattice effect on the bandgap. The lattice type mainly includes triangular lattice, square lattice, hexagonal lattice. We used triangular lattice. Fixed other parameters and only changing the lattice form for the triangle. Selected the shape of the scatterer for the cylindrical, scattering material for the lead. Scatter cylindrical radius was $0.0007 \mathrm{~m}$. The matrix material was the epoxy resin and lattice constant was fixed as $00017 \mathrm{~m}$.

The finite element method was used to analyze the first five characteristic frequencies and modes of phonon crystal structure. The results are shown in Table 4. In order to clearly observe the characteristics of the characteristics of the frequency, its mode is shown in Figure 6.

Table 4. Phonon Crystal Structure of the Original Cell Frequency Table

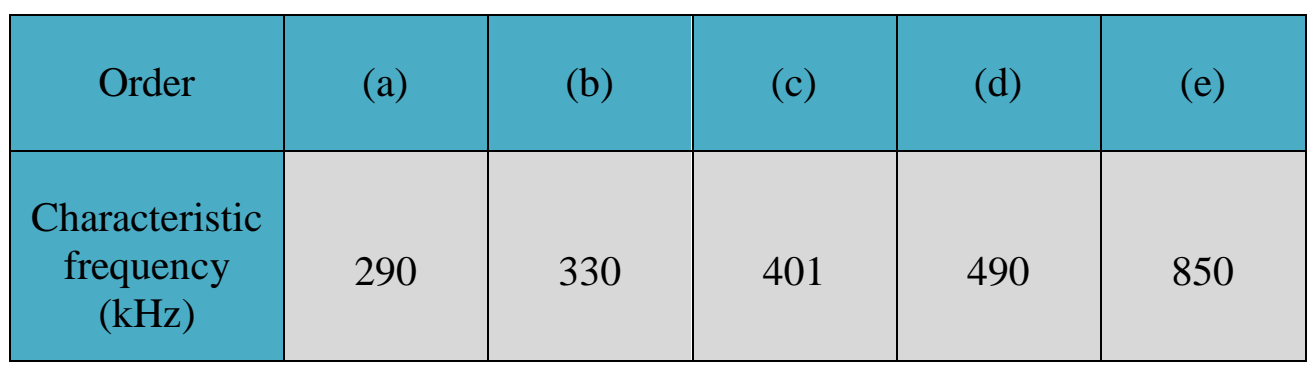

In Figure 6 (a), both the lead scatterer and the matrix vibrate. In Figure 6 (b) and (c) ,the vibration of the system is mainly concentrated in the matrix. The smaller the vibration of the lead scatterer is, the smaller amount of sound insulation and the narrower the bandgap is. Figure 6 (d) and (e)'s vibration are mainly concentrated in the vicinity of lead material and the substrate vibration is very small. The vibration displacement of the scatterer is reversed from that of the other four directions to cut off the original dispersion relation. So the sound transmission is small, which results in a wide bandgap of $490 \mathrm{kHz}$ to $850 \mathrm{kH}$. 

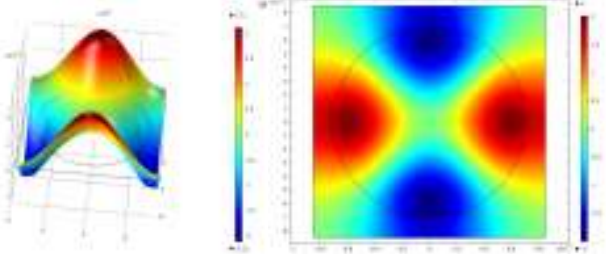

(a)

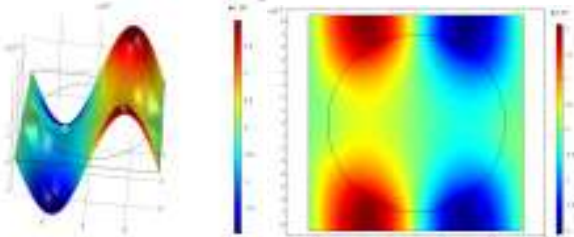

(c)

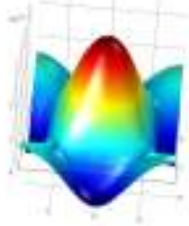

(e)
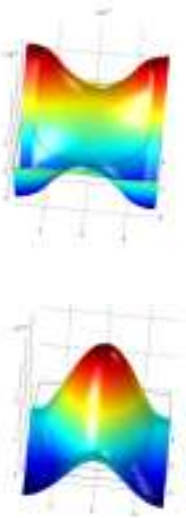

(b)

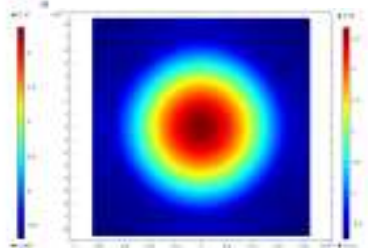

(d)

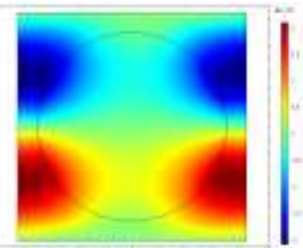

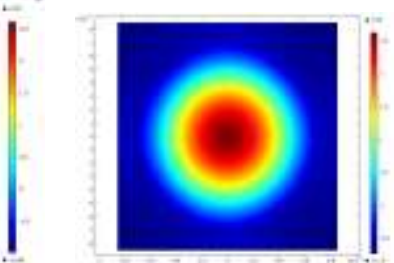

(e)

Figure 6. Finite Element Calculation Results of Triangular Lattice

In summary, when the scattering body shape is circular, scatterer material for the lead cylinder, scatterer cylindrical radius of $0.0007 \mathrm{~m}$, the base material for the epoxy resin, lattice constant fixed and only changing the lattice form for the triangular lattice, the bandgap width of the phononic crystal is widened. When the shape of the scatterer is closely arranged in a square lattice, its filling rate is 0.532 . When the triangular lattice is closely arranged, its filling rate is 0.614 . This is because in a more compact environment, the vibration of the scatterer and the interaction with the medium is more intense. Then we studied the effect of the fill rate on the bandgap.

\subsubsection{Effect of Filling Rate on the Structure of Phononic Crystal}

We studied the effect of the fill rate on the bandgap. Fixed the other parameters unchanged and changing only the fill rate. The lattice form selected the square lattice. The shape of the scatterer was circular. The scatterer materiala was lead cylinder. The matrix material was the epoxy resin, and the lattice constant a was $0.0017 \mathrm{~m}$.. We changed the filling rate by changing the radius of the lead cylinder. Lead cylindrical radius were taken $0.00051 \mathrm{~m}, 0.0007 \mathrm{~m}, 0.00074 \mathrm{~m}$ and so on. The corresponding filling rates were 0.321 . $0.532,0.643$ and so on. We used the finite element method to compute the bandgap structure and then obtained the following graph. 


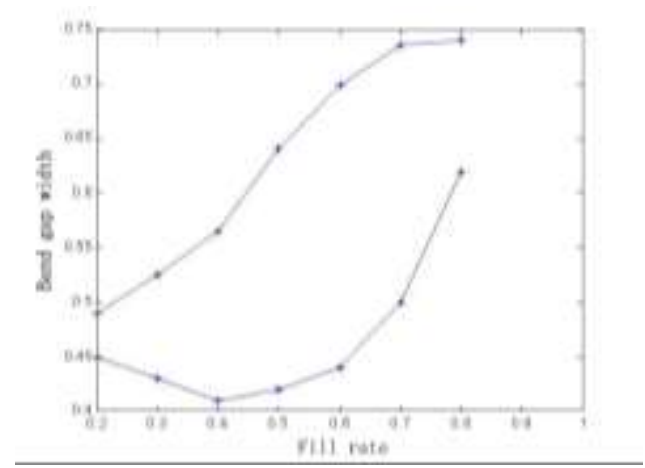

Figure 7. Variation of the Relative width of the Bandgap with the Filling Rate

It can be seen from Figure 7. that as the filling rate gradually increases, the band gap increases, the lower bound decreases gradually and the width becomes wider. This is because when the scatterer increases to a certain volume, the vibration effect between adjacent two columns is strengthened and the band gap width is increased. When the radius of the scatterer increases to $0.0007 \mathrm{~m}$ and the corresponding fill rate $\mathrm{f}$ is 0.532 , the lower boundary of the bandgap begins to move up and the corresponding bandgap width becomes smaller.

\subsection{Influence of Plate Thickness on Gap Structure}

The above discussion is based on a primitive to consider. However, in practical applications, we tend to apply it in a finite cycle. Therefore, it is necessary to research the vibration transfer characteristic of the phonon crystal of the periodic composite plate. Because of that the thickness $h$ is much smaller than the size of the xoy direction, so that the foregoing discussion, the effect of thickness on bandgap is ignored. But in real life, phonon crystals always have a certain thickness, which has a certain effect on the band gap. So the effect of the thickness of the phonon crystal plate on the bandgap is investigated.

The model used in this article is shown above, the lead, whose radius $r$ is 0.0007 meters, is arranged in the epoxy resin according to the $8 * 8$ cycle unit, its cross section is circular, the thickness $\mathrm{h}$ is $0.0015 \mathrm{~m}$, the lattice constant a is $0.0017 \mathrm{~m}$ and the fill rate $\mathrm{f}$ is 0.532 .

The analysis of the modal was carried out by finite element method. The first six characteristic frequencies and modal shape of phonon crystal structure were analyzed, and the results are shown in Table 5 below. In order to clearly observe the characteristics of the natural frequencies, the modal shape of each frequency is listed.

Table 5. Phonon Crystal Structure of the Original Cell Frequency Table

\begin{tabular}{|c|c|c|c|c|c|c|}
\hline Order & (a) & (b) & (c) & (d) & (e) & (f) \\
\hline $\begin{array}{c}\text { Characteristi } \\
\text { c frequency } \\
(\mathrm{kHz})\end{array}$ & 510 & 880 & 960 & 1010 & 1250 & 1250 \\
\hline
\end{tabular}

In order to study the characteristics of the phononic crystal plate more clearly, modal analysis of it was conducted. 


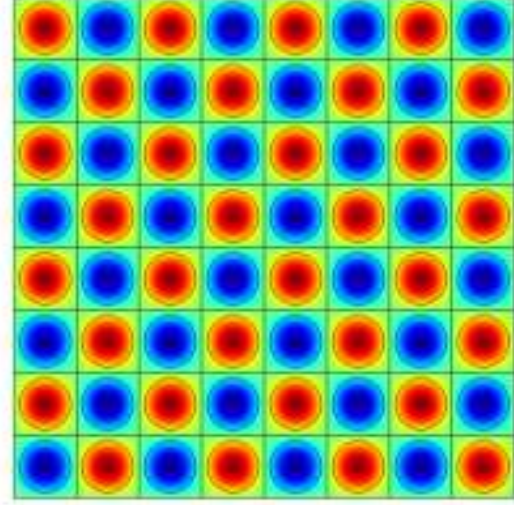

(a)

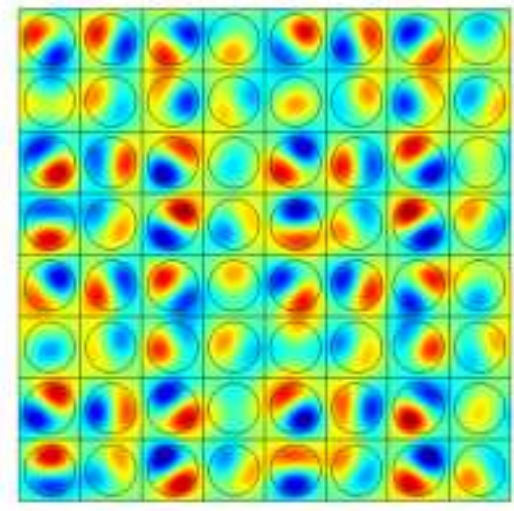

(c)

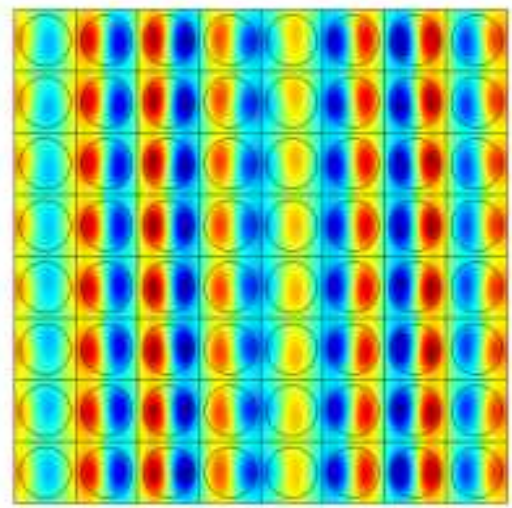

(e)

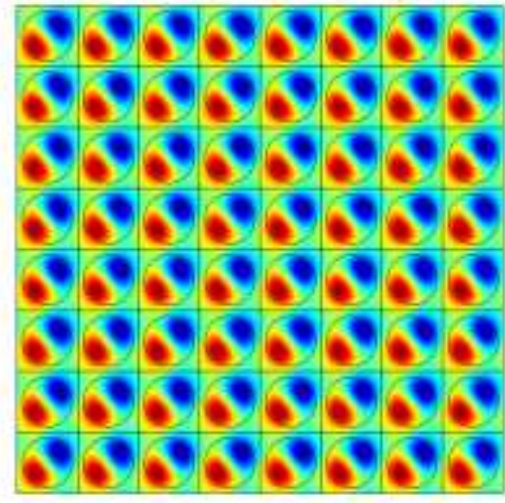

(b)

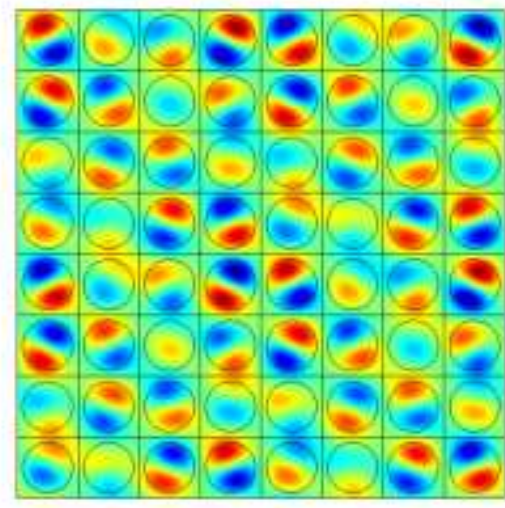

(d)

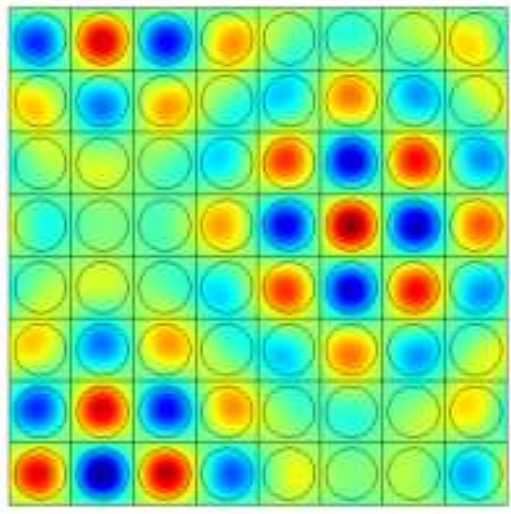

(f)

Figure 8. The Mode Diagram of the Phononic Crystal Plate under Different Modes

As shown in Figure 8, the vibrations in a single period in Fig. 8 (a) and (b) are all concentrated on the scatterers. The scatterer in Fig.8 (a) undergoes a total shear vibration. The vibrations of the substrate are small and the scatterer vibrates like a spring vibrator. The original dispersion curve is cut off. Vibrations in Fig.8 (b)are similar in a single cycle. The diagonal of the circle divides the scatterer cylinder into two semicircles. Two semicircles vibrate in opposite directions to form a band gap of $510 \mathrm{kHz} \sim 880 \mathrm{kHz}$. Fig. 8 (b), (c) and (e) are all different from each other in a single periodic unit, and it is difficult to form a band gap. Fig.8 (f) can not cause resonance because vibration is small. 
In studying the effect of the thickness of the phononic crystal plate on the bandgap, we only changed the thickness of the board and kept the other parameters unchanged. The thickness of the phonon crystal plate is $0.001 \mathrm{~m}, 0.0015 \mathrm{~m}, 0.002 \mathrm{~m}$ separately. The calculation is shown in Table 6.

Table 6. The Effect of Phonon Crystal Plate Thickness on Bandgap

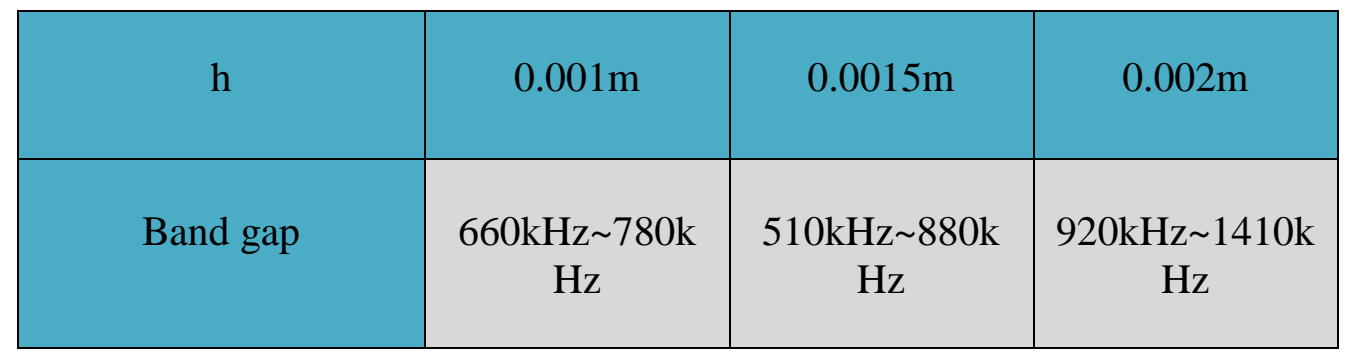

From the data in the table, as the thickness of the plate increases, the bandgap widens.

In practical applications, the board of the phonon crystal is thick, therefore, the effect of the thickness of the plate on the bandgap should be considered when designing the phonon crystal.

\section{Conclusion}

In this paper, we mainly discussed the bandgap structure of two-dimensional twoelement phononic crystals. Many factors influence the bandgap, which are divided into material parameters, structural parameters and the thickness of phononic crystal plate. In our study, the material parameters mainly included the parameters of the scatterer material and the material parameters of the matrix. The effects of the density, Young's modulus and shear modulus on the bandgap were discussed. It was concluded that when the scatterers and the matrix densities were quite different, the band gap width was wider and the material density mainly affected the lower boundary of the band gap. When the Young's modulus of the scatterer was large, the width of the band gap was wide, and the Young's modulus of the matrix had little effect on the band gap. The Young's modulus of the material mainly affected the upper boundary of the bandgap. The effect of the shear modulus of the scatterer and the matrix on the bandgap was small and could be negligible compared to the effect of the density and Young's modulus on the bandgap. Structural parameters mainly included lattice form, filling rate and scatterer shape. The lattice form took the triangular lattice and the square lattice, and it was concluded that only the lattice form was changed when the other parameters were determined, and the bandgap width of the triangular lattice was wider than that of the square lattice. The effect of the filling rate on the bandgap was that the bandgap width decreased as the filling rate increases. Moreover, The bandgap width became wider and increased to a certain extent. The thickness of the two-dimensional two-element phononic crystal plate had an impact on the band gap. That was to say, as the thickness of the plate increased, the band gap broadened in width, which provided theoretical guidance for the actual design of phononic crystals.

\section{Acknowledgments}

This project was sponsored in part by National Natural Science Foundation of China (No.61671414), Natural Science Foundation for Young Scientists of Shanxi Province, China (No.201601D202035) and China Postdoctoral Science Foundation (2017M611198). 


\section{References}

[1] C. Qiu, Z. Liu, J. Mei, and J. Shi, "Mode-selecting acoustic filter by using resonant tunneling of twodimensional double phononic crystals",Appl. Phys. Lett., vol. 87, no.10, (2005), pp. 2059.

[2] M. J. Frazier and M. I. Hussein, "Viscous-to-viscoelastic transition in phononic crystal and metamaterial band structures", The Journal of the Acoustical Society of America, vol. 138, no.5, (2015), pp.3169-3180.

[3] A. A. Kutsenko, A. L. Shuvalov, A. N Norris, and O. Poncelet,, "Effective shear speed in twodimensional phononic crystals", Physical Review B, vol. 84, no.6, (2011), pp. 7615-7619.

[4] Y. Z. Wang, F. M. Li, K. Kishimoto, Y. S. Wang, and W. H. Huang, "Wave localization in randomly disordered layered three-component phononic crystals with thermal effects", Archive of Applied Mechanics, vol. 80, no.6, (2010), pp. 629-640.

[5] F. L. Li, Y. S. Wang and C. Zhang, "Bandgap calculation of two-dimensional mixed solid-fluid phononic crystals by dirichlet-to-neumann maps", Physica Scripta, vol. 84, no.5, (2011), pp. 055402.

[6] Z. Liu, C. T. Chan and P. Sheng. "Analytic model of phononic crystals with local resonances", Physical Review B, vol. 71, no.1, (2005), pp. 014103(1-8).

[7] X. Zhang, Y. Liu, F. Wu and Z. Liu, "Large two-dimensional band gaps in three-component phononic crystals", Physics Letters A, vol. 317, no.1, (2003), pp. 144-149.

[8] X. P. Wang, P. Jiang, T. N. Chen and K. P. Yu, "Frequency characteristics of defect states in a twodimensional phononic crystal with slit structure", International Journal of Modern Physics B, vol. 30, no.6, (2016), pp. 1650025.

[9] Maasilta, I. J., Puurtinen, T. A., Tian, Y and Geng, Z, "Phononic thermal conduction engineering for bolometers: from phononic crystals to radial casimir limit", Journal of Low Temperature Physics, vol. 184, no. 1, (2016), pp. 211-216.

[10] C. Gu and F. Jin, "Research on the tunability of point defect modes in a two-dimensional magnetoelastic phononic crystal", Journal of Physics D-Applied Physics, vol. 49, no. 17, (2016), pp. 175103.

[11] P. Ji, W. Hu and J. Yang, "Development of an acoustic filter for parametric loudspeaker using phononic crystals", Ultrasonics, vol. 67, no. 10, (2016), pp. 160-167.

[12] M. Haras, V. Lacatena, T. M. Bah, S. Didenko, J. F. Robillard and S. Monfray, "Fabrication of thin-film silicon membranes with phononic crystals for thermal conductivity measurements", IEEE Electron Device Letters, vol. 37, no. 10, (2016), pp. 1358-1361.

[13] H. W. Dong, X. X. Su, Y. S. Wang and C. Zhang, "Topology optimization of two-dimensional asymmetrical phononic crystals", Physics Letters A, vol. 378, no. 4, (2014), pp. 434-441.

[14] H. W. Dong, X. X. Su and Y. S. Wang, "Multi-objective optimization of two-dimensional porous phononic crystals", Journal of Physics D Applied Physics, vol. 47, no. 15, (2014), pp. 494-500.

[15] N. Aravantinoszafiris, M. M. Sigalas, M. Kafesaki, and E. N. Economou, "Phononic crystals and elastodynamics: some relevant points", Aip Advances, vol. 4, no. 12, (2014), pp. 1492-382.

[16] X. X. Su, Y. F. Wang, and Y. S. Wang, "Effects of Poisson's ratio on the band gaps and defect states in two-dimensional vacuum/solid porous phononic crystals", Ultrasonics, vol. 52, no. 2, (2012), pp. 255.

[17] Y. Cao, Z. Hou, W. Sritrakool and Y. Liu, "Reflection properties and effective parameters of twodimensional phononic crystals", Physics Letters A, vol. 337, no. 1, (2005), pp. 147-154.

[18] J. O. Vasseur, M. Beaugeois, B. Djafari-Rouhani, Y. Pennec and P. A. Deymier, "Transmission of acoustic waves through waveguide structures in two-dimensional phononic crystals", Physica Status Solidi, vol. 1, no. 11, (2004), pp. 2720-2724.

[19] W. Nan, M. L. Tsai, F. L. Hsiao, W. S. Bo, D. L. Kwong and M. Palaniapan, "Micromechanical resonators based on silicon two-dimensional phononic crystals of square lattice", Journal of Microelectromechanical Systems,vol. 21, no. 4, (2012), pp. 801-810.

[20] M. J. Frazier and M. I. Hussein, "Generalized Bloch's theorem for viscous metamaterials: Dispersion and effective properties based on frequencies and wavenumbers that are simultaneously complex", Comptes rendus - Physique, vol. 17, no. 5, (2016), pp. 565-577. 
International Journal of Advanced Science and Technology

Vol.110 (2018) 